\title{
Analyzing thermal convection in a two-dimensional circular annulus via spatio-temporal Koopman decomposition
}

\author{
Juan Sánchez Umbría a , Marta Net a , José M. Vega b,* \\ a Departament de Física, Universitat Politècnica de Catalunya, 08034 Barcelona, Spain \\ ${ }^{\mathrm{b}}$ E.T.S.I. Aeronáutica y del Espacio, Universidad Politécnica de Madrid, 28040 Madrid, Spain
}

\section{A R T I C L E I N F O}

\section{Article history:}

Received 27 February 2019

Received in revised form 29 October 2019

Accepted 31 October 2019

Available online $\mathrm{xxxx}$

Communicated by T. Sauer

\section{Keywords:}

Spatio-temporal Koopman decomposition

Nonlinear dynamical systems

Hydrodynamic instabilities

Thermal convection in a two-dimensional

circular annulus

Spatio-temporal dynamics

Symmetries

Periodic and quasi-periodic dynamics

Invariant tori

\begin{abstract}
A B S T R A C T
This paper deals with the analysis of numerically obtained spatio-temporal data for thermal convection in a two-dimensional circular annulus. These data are post-processed using a recent method called the spatio-temporal Koopman decomposition, which applies to the frequent case in which the underlying dynamics exhibit oscillatory (possibly growing or decaying) behavior in both time and one of the spatial directions (the azimuthal coordinate for the present problem). When this holds, the method decomposes the data into Fourier-like series in both the distinguished spatial direction and time. In the general case, the obtained series account for, not only the involved temporal frequencies and spatial wavenumbers, but also the spatial and temporal growth rates. In the simpler situation of attractors showing spatially periodic behavior, the spatial and temporal growth rates vanish (or are very small). In this case, the analysis of the wavenumber-frequency pairs that are present allows for uncovering the spatio-temporal structure of the flow in the circular annulus. The analysis focuses on periodic and quasi-periodic attractors, which exhibit spatio-temporal symmetries that are identified by the method. In addition, for quasi-periodic attractors, the method gives semi-analytic descriptions for the tori densely covered by particular trajectories. Although the paper concentrates on the thermal convection problem in an annulus, it will become clear that the method applies to other related dynamics as well.
\end{abstract}

(C) 2019 Elsevier B.V. All rights reserved.

\section{Introduction}

Many, generally nonlinear dynamical systems are such that the temporal dynamics of the scalar or vector state variable $q^{s}$ (with the superscript $s$ denoting the components of the state variable) depending on the vector spatial coordinates $\boldsymbol{x}$ and time $t$, exhibit the following approximate behavior

$q^{S}(\boldsymbol{x}, t)=\sum_{n=-N}^{N} a_{n} u_{n}^{s}(\boldsymbol{x}) \mathrm{e}^{\left(\delta_{n}+\mathrm{i} \omega_{n}\right) t}$,

where $a_{n} \geq 0$ and $u_{n}^{s}$ are the mode amplitudes and the conveniently normalized spatial modes, respectively, and $\delta_{n}$ and $\omega_{n}$ are the temporal growth rates and frequencies, respectively. For attractors, the growth rates $\delta_{n}$ are all zero (or very small). Positive values of $\delta_{n}$ are associated to instabilities and negative values, to transient behavior approaching attractors. Examples are numerous and include, e.g., purely fluid dynamic problems, such as vortex shedding [1], aeroelastic phenomena [2], and thermal

\footnotetext{
* Corresponding author.
}

E-mail addresses: juan.j.sanchez@upc.edu (J.S. Umbría), marta.net@upc.edu (M. Net), josemanuel.vega@upm.es (J.M. Vega). convection problems $[3,4]$ in $2 \mathrm{D}$ or $3 \mathrm{D}$. Note that, strictly speaking, the = sign appearing in Eq. (1.1) (and in many equations below) should be replaced by $\sim e q$ for two main reasons. First, the data in the left hand side are usually subject to errors. Second, the expansion in the right hand side is truncated retaining only $2 N+1$ terms. However, Eq. (1.1) can be considered as exact to the approximation relevant in this paper and the = sign will be maintained hereinafter. The involved errors will be indicated.

If the data are real, as we assume hereinafter in this paper, those terms in (1.1) can be conformed in complex conjugate pairs, which means that the $2 N+1$ terms appearing in the expansion (1.1) can be organized such that they are invariant under the action

$n \rightarrow-n, \quad \omega_{n} \rightarrow-\omega_{n}, \quad u_{n}^{s} \rightarrow \bar{u}_{n}^{s}$,

where the overbar stands hereinafter for the complex conjugate. Note that when the components of the state vector are coupled, $\delta_{n}$ and $\omega_{n}$ are typically common to the state variable components, namely they are independent of $s$. The expansion (1.1) will be obtained in discretized form as

$q^{S}\left(\boldsymbol{x}_{i}, t_{k}\right)=\sum_{n=-N}^{N} a_{n} u_{n}^{s}\left(\boldsymbol{x}_{i}\right) \mathrm{e}^{\left(\delta_{n}+\mathrm{i} \omega_{n}\right) t_{k}}$, 
where the discrete values of time, $t_{k}$, must be equispaced, which is a very important requirement for the methods used below. The expansion (1.3) can be computed using standard dynamic mode decomposition (DMD) [5]. However, as further explained in [6], this method fails when the spatial complexity (defined as the rank of the set of spatial modes) is smaller than the spectral complexity (defined as the number of terms that are present in the expansion). In this case, standard DMD can be substituted by a recent extension, called higher order DMD (HODMD) [6], which will be summarized Section 2 and gives good results for arbitrary values of the spatial and spectral complexities [6]. The method depends on three tunable parameters, but at the moment we only mention a (small) threshold $\varepsilon_{2}$ that defines truncation. Namely, we only retain those modes such that

$a_{n} / a_{\max } \geq \varepsilon_{2}$,

where $a_{\max }$ is the maximum value of the mode amplitudes. This truncation condition defines $N$ in the expansions (1.1) and (1.3).

Whatever the method, setting $t_{k}$ continuous in the expansion (1.3) leads to temporal interpolation. Spatial interpolation in $\boldsymbol{x}$ (which is only known at the considered discrete values of $\boldsymbol{x}$ associated with the spatial grid) could also be performed using an appropriate interpolation method. On the other hand, for transient dynamics converging to an attractor, some of the modes are such that $\delta_{n}=0$ (or very small) and the remaining ones exhibit negative $\delta_{n}$. Eliminating from the expansion the latter modes permits computing the attractor (temporal extrapolation [7]). In the sequel, we concentrate on attractors, for which the temporal growth rates are all zero (or conveniently small). In this case, a good account of how well the expansions (1.1) and (1.3) converge results from a semi-logarithmic plot of the amplitudes vs. the frequencies, which will be called the $a-\omega$ diagram. Time may be replaced in Eqs. (1.1) and (1.3) by a distinguished spatial coordinate, which gives a spatial counterpart of these decompositions, called spatial DMD.

When the modes appearing in Eqs. (1.1) and (1.3) are proportional to their complex conjugates, namely $\bar{u}_{n}^{s}=\mathrm{e}^{\mathrm{i} \alpha^{s}} u_{n}^{s}$, the state variable oscillates in phase at all spatial points, giving a pure standing wave (SW), which appear for instance when the dynamics exhibit a convenient instantaneous reflection symmetry. Note that the phase $\alpha^{s}$ does generally depend on $s$ because a phase shift between the various state variable components is generally to be expected. When the phases also depend on $n$, the expansion (1.3) gives a modulated $S W$, in which the pattern goes not only up and down but also, say, left and right as time proceeds. These patterns appear generically when the dynamics exhibit a spatio-temporal reflection symmetry. Examples of pure and modulated SWs will be given below. More general, nonstanding patterns are progressive, and may show a very complex dynamical structure or not. Among the latter, for systems that are invariant under translations in a distinguished direction, $x$, pure traveling waves (TWs) are steady in axes moving along the longitudinal direction $x$ and modulated TWs are generally periodic or quasi-periodic in moving axes. TWs (and also SWs, see the applications in this paper) are more clearly analyzed using the following spatio-temporal counterpart of Eq. (1.1)

$q^{s}(\boldsymbol{y}, x, t)=\sum_{m=-M}^{M} \sum_{n=-N}^{N} a_{m n} u_{m n}^{s}(\boldsymbol{y}) \mathrm{e}^{\left(\nu_{m}+\mathrm{i} \kappa_{m}\right) x+\left(\delta_{n}+\mathrm{i} \omega_{n}\right) t}$

where vector $\boldsymbol{y}$ denotes the remaining transverse spatial coordinates, $v_{m}$ and $\kappa_{m}$ are the spatial growth rates and wavenumbers, respectively, and the spatio-temporal amplitudes and normalized modes are $a_{m n} \geq 0$ and $u_{m n}^{s}$, respectively. As above, the discrete counterpart of this expansion is

$q^{s}\left(\boldsymbol{y}_{i}, x_{j}, t_{k}\right)=\sum_{m=-M}^{M} \sum_{n=-N}^{N} a_{m n} u_{m n}^{s}\left(\boldsymbol{y}_{i}\right) \mathrm{e}^{\left(\nu_{m}+\mathrm{i} \kappa_{m}\right) x_{j}+\left(\delta_{n}+\mathrm{i} \omega_{n}\right) t_{k}}$, where the discrete values of $x$ and $t, x_{j}$ and $t_{k}$, respectively, are both taken as equispaced, which again is a very important condition for the methods in this paper. And, again, setting both $x_{j}$ and $t_{k}$ continuous involves interpolation in these variables; a further convenient interpolation in $\boldsymbol{y}$ leads to Eq. (1.5).

Since the state variable is real, the various terms in the expansions (1.5) and (1.6) appear in complex conjugate pairs, which means that they may be organized such that they are invariant under the action

$m \rightarrow-m, \quad n \rightarrow-n, \quad \kappa_{m} \rightarrow-\kappa_{m}, \quad \omega_{n} \rightarrow-\omega_{n}$,

$u_{m n}^{s} \rightarrow \bar{u}_{m n}^{s}$,

which is the counterpart of Eq. (1.2).

The expansion in (1.6) will be obtained via the spatio-temporal Koopman decomposition (STKD) method [8], which will be summarized in Section 2. As in the purely temporal decomposition (1.3), a tunable parameter $\varepsilon_{2}$ is used to truncate (1.6), retaining only those spatio-temporal modes such that ( $\mathrm{cf}(1.4)$ )

$a_{m n} / a_{\max } \geq \varepsilon_{2}$,

where $a_{\max }$ is the maximum value of the mode amplitudes. This truncation condition defines both $M$ and $N$, which in principle define the total number of modes appearing in (1.5) and (1.6). However, typically, condition (1.8) leads to a smaller number of modes to be retained, namely $P \leq(2 M+1)(2 N+1)$. The resulting expansion can still be written in the form (1.6), with the neglected amplitudes set equal to zero.

When the dynamics correspond to a temporal attractor whose spatial structure is also permanent in the spatial variable $x$, the spatial and temporal growth rates are very small. In this case, the plot of $\kappa$ vs. $\omega$, to be called the $\kappa-\omega$ diagram, will give an idea of the structure of the dynamics, identifying in particular SWs or TWs. In the former case, such diagram conforms horizontal lines, while in the latter case it is organized in oblique straight lines. For thermal convection in a 2D annular geometry, SWs will be analyzed in the applications along the paper, while examples of TWs appearing in both the 1D Ginzburg-Landau equation and thermal convection in a 3D spherical shell can be found in [8].

It is to be noted that the preliminary applications in [8] were only intended to illustrate the performance of the STKD method. Here, instead, we use the method to analyze the dynamics for thermal convection in a two-dimensional circular annulus, for which we perform a deeper analysis of periodic and quasi-periodic attractors, focusing on the identification of the symmetries that are present in the dynamics. Also, for quasiperiodic attractors, we obtain a semi-analytic description of the whole torus densely covered by the particular trajectories. It is remarkable that efficiently identifying invariant tori associated with quasi-periodic solutions in high-dimensional phase spaces is a major open problem in the field; in the applications below, the quasi-periodic attractors will exhibit only two incommensurable fundamental frequencies, but it will become plain clear that the method is straightforwardly extended to higher dimensional tori. Also, we anticipate that these results will be obtained using data in a limited timespan, which makes a significant difference with more standard methods, such as FFT and PSD [9]. Other known methods are available to obtain the frequencies and amplitudes for scalar signals (instead of the high-dimensional signals considered in this paper). These methods use relatively short times, and are based on iterative procedures $[10,11]$. The particular problem considered here is used for the sake of clarity in the exposition, but it will become clear that the ideas in the paper (in connection with identifying both symmetries and invariant tori) also apply to other thermal convection problems and, moreover, to other dynamical systems. 
The truncated expansions considered above are only approximations of the actual dynamics. The error of these approximations will be measured in terms of the relative root mean square (RMS) error, defined for the discrete approximations as

RRMS error $=\frac{\left\|q_{\text {approx. }}^{s}-q_{\text {exact }}^{s}\right\|_{2}}{\left\|q_{\text {exact }}^{s}\right\|_{2}}$,

where the norm $\|\cdot\|_{2}$ denotes hereinafter the Euclidean norm for vectors and the Frobenius norm for matrices or tensors.

Note that expansions of the type (1.1) and (1.5) (usually retaining only a few terms) are implicit in the weakly-nonlinear analysis of oscillatory pattern forming systems [12-15], using approximations of the governing equations. The methods considered in this paper, instead, give these expansions for fully nonlinear dynamics in a purely data-driven fashion, without using at all the model equations that govern the underlying dynamics.

The remaining of the paper is organized as follows. Section 2 summarizes the temporal HODMD and spatio-temporal STKD methods. In Section 3, the STKD method is applied to the thermal convection in a 2D annulus. Three cases are considered. The first two correspond to periodic solutions in time and the third one, to a quasi-periodic regime. The case of noisy data is considered in Section 4. The article ends with some conclusions and remarks, in Section 5.

\section{The HODMD and STKD methods}

Let us now give a brief description of the HODMD and STKD methods.

The temporal HODMD method yields the expansion (1.3) using a set of $K$ snapshots that can be organized in a snapshot matrix, whose elements are given by $q^{s}\left(\boldsymbol{x}_{i}, t_{k}\right)$. The method proceeds in three steps that are summarized here (see [6] for more details and the relevant formulae to compute the expansion and see [16] for a MATLAB executable): (a) the first step consists in a dimension reduction, which decreases the approximate rank of the snapshots set using a truncated singular value decomposition (SVD) [17] applied to the snapshot matrix. This step takes advantage of the redundancies among the snapshots and truncation is performed using a tunable tolerance $\varepsilon_{1}$. The outcome is a set of dimension-reduced snapshots and an inverse projection matrix that permits reconstructing the original snapshots from the dimension-reduced snapshots. (b) As a second step, each dimension-reduced snapshot is enlarged considering also the former $d-1$ (with $d \geq 1$ tunable) time-shifted snapshots and the standard DMD method is applied to these enlargedreduced snapshots, which leads to a dimension-reduced version of the expansion (1.3); the mode amplitudes are computed via least squares fitting with the actual reduced snapshots. The expansion (1.3) is recovered from its dimension-reduced version using the inverse projection matrix mentioned in step (a) and appropriate rescaling the resulting spatial modes (which leads to a re-definition of the mode amplitudes computed for the dimension-reduced expansion). (c) As a third step, the resulting expansion (1.3) is truncated according to (1.4) (with $\varepsilon_{2}$ tunable), which defines the number of retained modes, $2 N+1$. Note that this algorithm, called the DMD-d algorithm, synergically combines the advantages of standard DMD and the Takens' delayed embedding theorem [18].

As described, the method depends on (i) the timespan in which the method is applied, which should be somewhat larger (say, 1.5 times as large) than the largest involved period, (ii) the number of equispaced snapshots taken in this timespan, which should be such that the distance between consecutive values of $t$ is much smaller (say, five times smaller) than the smallest involved period, and (iii) the spatial discretization used in the variable $\boldsymbol{x}$. In addition, the method depends on three tunable parameters, namely $\varepsilon_{1}$ (defining truncation in step (a)), $\varepsilon_{2}$ (defining truncation in step (c)), and $d$ (defining the number of delays in step (b)). It is to be noted that the selection of $d$ is not critical, namely the plot of the error of the approximation vs. $d$ is usually fairly flat near the minimum [6] and, for a given timespan, the number of delays, $d$, scales with the number of snapshots $K$. Robustness of the results against variations of the various parameters is an important property that must be checked in the application of the method. When the index $d=1$, the HODMD method described above reduces [6] to the standard DMD method [5].

Likewise, spatial DMD along a distinguished spatial coordinate $x$ is performed similarly, by just substituting $t$ by $x$.

Now, the STKD method gives the spatio-temporal expansion (1.6), whose left hand side depends on three indexes and thus defines a snapshot tensor, instead of a snapshot matrix. The method combines higher order SVD (HOSVD), a method introduced by Tucker [19] and more recently popularized by de Lathauwer et al. [20], and two applications of HODMD performed along the longitudinal and temporal directions, $x$ and $t$, respectively, with indexes $d^{x} \geq 1$ and $d^{t} \geq 1$, respectively, which do not necessarily coincide; see [8] for more details and the relevant formulae. Truncation in the application of the HOSVD method (which can be seen as a dimension reduction) is performed requiring that the relative RMS error of the truncated approximation be smaller that some (small) tunable parameter $\varepsilon_{1}$. This is a good strategy when the data are very clean. However, for noisy data, truncation can be performed (to filter noise) in view of the HOSVD singular values distribution (see Section 4).

Concerning the computational cost of the STKD method, note that it requires [8]: (a) applying tools such standard SVD and HOSVD, which reduce the dimension of the snapshot matrix or tensor, (b) computing eigenvalues of some dimension-reduced matrices, to calculate the spatial and temporal growth rates, wavenumbers, frequencies, and modes, and (c) applying a dimension reduced least squares fitting to compute the mode amplitudes. These operations require polynomial times in the amount of involved data. For the specific case considered in this paper, the most computationally expensive STKD computation (for the quasiperiodic dynamics considered below) required $\sim 24 \mathrm{CPU}$ seconds in a standard PC, with a microprocessor Intel Core $17-8700 \mathrm{~K}$ at $3.7 \mathrm{GHz}$.

\section{Thermal convection in a two-dimensional annulus}

Let us analyze some periodic and quasi-periodic solutions obtained in the study of the thermal convection in an annulus subject to an inward radial gravity $g^{*}$, and heated from the inside. The inner and outer radii are $r_{i}^{*}$ and $r_{o}^{*}$, respectively, with the superscript ${ }^{*}$ denoting hereinafter dimensional quantities. The problem is nondimensionalized using $r_{o}^{*}-r_{i}^{*},\left(r_{o}^{*}-r_{i}^{*}\right)^{2} / \kappa^{*}$, and $\Delta T^{*}=T^{*}\left(r_{i}^{*}\right)-T^{*}\left(r_{0}^{*}\right)$ as units for length, time, and temperature, respectively. In the Boussinesq approximation, the problem depends on three nondimensional parameters, the radius ratio $\eta=r_{i}^{*} / r_{0}^{*}$, and the Rayleigh and Prandtl numbers, defined as

$R a \equiv \frac{\alpha^{*} \Delta T^{*} g^{*}\left(r_{o}^{*}-r_{i}^{*}\right)^{3}}{\kappa^{*} v^{*}}, \quad \operatorname{Pr} \equiv \frac{\nu^{*}}{\kappa^{*}}$,

where $\kappa^{*}$ is the thermal diffusivity, $v^{*}$ is the kinematic viscosity, and $\alpha^{*}$ is the thermal expansion coefficient.

The annulus is described in terms of the nondimensional radial, azimuthal, and temporal variables, $r, \phi$, and $t$, respectively. The continuity, Navier-Stokes, and energy equations are formulated in terms of a streamfunction, $\psi(r, \phi, t)$, the azimuthal average of the azimuthal velocity, $f(r, t)$, and the perturbation 
of the temperature, $\Theta(r, \phi, t)$, with respect to the quiescent conduction profile, given by $\boldsymbol{v}=\mathbf{0}, T=T_{c}(r)$. Thus, the continuity equation is identically fulfilled and the nondimensional temperature and velocity fields are written as

$T=T_{c}+\Theta, \quad \mathbf{v}=f \hat{\phi}+\nabla \times(\psi \hat{k})$,

where $\hat{k}$ is the unit vector normal to the plane containing the annulus. The function $f(r, t)$ is needed to guarantee the possible existence of a non-zero azimuthal average of the azimuthal velocity, which is zero if homogeneous boundary conditions are taken for $\psi$. Thus, actually, the total streamfunction is

$\Psi(r, \phi, t)=\psi(r, \phi, t)-\int_{r_{i}}^{r} f(r, t) d r$.

The governing equations are

$$
\begin{aligned}
\left(\partial_{t}-\operatorname{Pr} \tilde{\Delta}\right) f= & P_{\phi}\left[\Delta \psi\left(\frac{1}{r} \partial_{\phi} \psi\right)\right], \\
\left(\partial_{t}-\operatorname{Pr} \Delta\right) \Delta \psi= & \frac{\operatorname{Pr} \operatorname{Ra}}{r} \partial_{\phi} \Theta+\left(1-P_{\phi}\right) J(\psi, \Delta \psi)+ \\
& \tilde{\Delta} f\left(\frac{1}{r} \partial_{\phi} \psi\right)-f\left(\frac{1}{r} \partial_{\phi} \Delta \psi\right), \\
\left(\partial_{t}-\Delta\right) \Theta= & -\frac{1}{r^{2} \ln \eta} \partial_{\phi} \psi+J(\psi, \Theta)-f\left(\frac{1}{r} \partial_{\phi} \Theta\right),
\end{aligned}
$$

where $P_{\phi}$ is the operator that averages in the azimuthal direction, $\Delta$ is the horizontal Laplacian operator in polar coordinates, $J(g, h)=\left(\partial_{r} g \partial_{\phi} h-\partial_{r} h \partial_{\phi} g\right) / r$, and $\tilde{\Delta}=\partial_{r}\left(\partial_{r}+1 / r\right)$. The non-slip and constant temperature boundary conditions become

$f=\psi=\partial_{r} \psi=\Theta=0 \quad$ on $r=r_{i}, r_{0}$.

The above system is $\mathcal{O}(2)$ equivariant, i.e., invariant under arbitrary azimuthal rotations $R_{\phi_{R}}$

$R_{\phi_{R}}: \quad(f, \psi, \Theta)(r, \phi, t) \rightarrow(f, \psi, \Theta)\left(r, \phi+\phi_{R}, t\right)$,

and reflections $\zeta_{\phi_{0}}$ through any angle $\phi=\phi_{0}$

$\zeta_{\phi_{0}}: \quad(f, \psi, \Theta)\left(r, \phi_{0}+\phi, t\right) \rightarrow(f,-\psi, \Theta)\left(r, \phi_{0}-\phi, t\right)$.

To obtain the data that will be used below, the fields $\psi$ and $\Theta$ are discretized using a Fourier expansion up to order 192 in the azimuthal coordinate, $\phi$, and collocation, on a Gauss-Lobatto mesh of 32 points, in the radial coordinate $r$. The resulting set of equations is integrated in time by using a fourth order semiimplicit BDF-extrapolation time-stepping scheme with a fixed time step $\Delta t=10^{-4}$. With this discretization, the relative RMS truncation error is $\sim 10^{-9}$ (see [21]). However, it is important to note that the discretization method is irrelevant for the application of the HODMD and STKD methods. The only requirement is that the pointwise data be equispaced in both the azimuthal coordinate and time. With this very small discretization error and the large amount on spatial data, the DMD-1 algorithm (namely, the standard DMD method) will provide good results. However, for not so clean data, the index $d$ in the DMD- $d$ algorithm must be taken larger than one, as it will be seen in Section 4 .

In order to apply the HODMD and STKD methods below, a set of $K$ snapshots, equispaced in time, is selected in an appropriate time interval. Each snapshot will contain the values of $\Psi$ and $\Theta$ on a mesh of $I$ points in $r$ and $J$ equispaced points in $\phi$. The HODMD and STKD methods can be applied to both $\Psi$ and $\Theta$ simultaneously, or separately. In the former case, some care must be taken with the order of magnitude of both variables depending on the nondimensionalization choosen, meaning that some scaling could be necessary before applying the methods. Here, for simplicity, we shall apply the methods to only the temperature. Results for the streamfunction are similar and omitted.
The HODMD method can be applied either in space or time, which leads to the expansions

$\Theta(r, \phi, t)=\sum_{m=-M}^{M} a_{m}^{\text {spatial }} u_{m}^{\text {spatial }}(r, t) \mathrm{e}^{\left(\nu_{m}+\mathrm{i} \kappa_{m}\right) \phi}$

and

$\Theta(r, \phi, t)=\sum_{n=-N}^{N} a_{n}^{\text {temporal }} u_{n}^{\text {temporal }}(r, \phi) \mathrm{e}^{\left(\delta_{n}+\mathrm{i} \omega_{n}\right) t}$,

respectively, where the spatial and temporal modes, $u_{m}^{\text {spatial }}$ and $u_{n}^{\text {temporal }}$, are conveniently normalized to exhibit unit RMS, as

$\frac{\left\|u_{m}^{\text {spatial }}\right\|_{2}}{\sqrt{I K}}=1, \quad \frac{\left\|u_{n}^{\text {temporal }}\right\|_{2}}{\sqrt{I J}}=1$.

This RMS normalization defines the spatial and temporal amplitudes $a_{m}^{\text {spatial }} \geq 0$ and $a_{n}^{\text {temporal }} \geq 0$, respectively, which permits truncation according to (1.4). However, these expansions give a less enlightening information about the spatio-temporal structure of the dynamics than the spatio-temporal expansion (obtained via the STKD method)

$\Theta(r, \phi, t)=\sum_{m=-M}^{M} \sum_{n=-N}^{N} a_{m n} u_{m n}(r) \mathrm{e}^{\left(v_{m}+\mathrm{i} \kappa_{m}\right) \phi+\left(\delta_{n}+\mathrm{i} \omega_{n}\right) t}$,

where the spatio-temporal modes are conveniently normalized to exhibit unit RMS, as

$\frac{\left\|u_{m n}\right\|_{2}}{\sqrt{I}}=1$

which defines the amplitudes $a_{m n} \geq 0$ and permits truncating the expansion according to (1.8). It is convenient to note that, to the approximation relevant here, the spatial and temporal expansions (3.10) and (3.11) are readily obtained from the spatio-temporal expansion (3.13) as

$a_{m}^{\text {spatial }} u_{m}^{\text {spatial }}(r, t)=\sum_{n=-N}^{N} a_{m n} u_{m n}(r) \mathrm{e}^{\left(\delta_{n}+\mathrm{i} \omega_{n}\right) t}$,

$a_{n}^{\text {temporal }} u_{n}^{\text {temporal }}(r, \phi)=\sum_{m=-M}^{M} a_{m n} u_{m n}(r) \mathrm{e}^{\left(\nu_{m}+\mathrm{i} \kappa_{m}\right) \phi}$.

Recall that $u^{\text {spatial }}$ and $u^{\text {temporal }}$ must be rescaled in these equations such that (3.12) holds.

In the remaining of this section, we consider three cases exhibiting different spatio-temporal symmetries, namely a simple periodic flow, a more complex periodic flow, and a quasi-periodic flow. In all cases, the numerical solver is first run along a time stage to eliminate transient behavior in which the dynamics approach the considered time-dependent attractors; snapshots are collected after this time stage, whose length depends, in general, on several factors. The most important of these are the initial conditions, the degree of stability of the attractor, and the presence of nearby bifurcations or other branches of solutions, such that the transient trajectory could spend long times near weakly unstable solutions. In the present problem, to reach stable saturated periodic solutions, takes less than 2 time units if the initial condition is a nearby periodic attractor. This transient stage is approximately 14 times larger than the timespan where the snapshots are sampled. For the quasi-periodic solutions, the transient takes less than 15 time units, which is 3 times larger than the timespan where the snapshots are collected. In all cases, we ensure that after this transient stage we are already in the attractor by checking that the temporal growth rates of the calculated modes are all very small. The outcomes of the STKD method will 
(a)

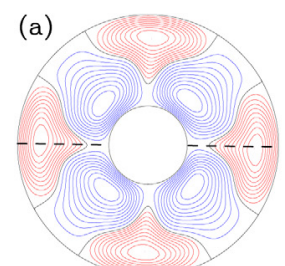

(e) (b)

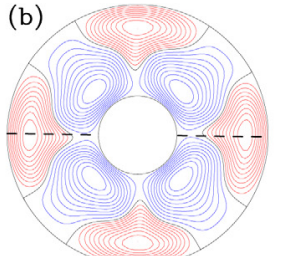

(f)

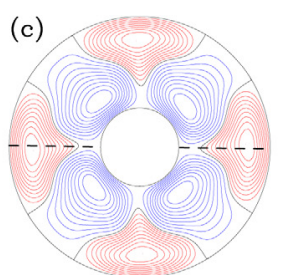

(g)

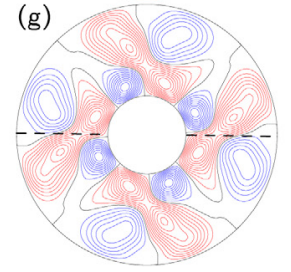

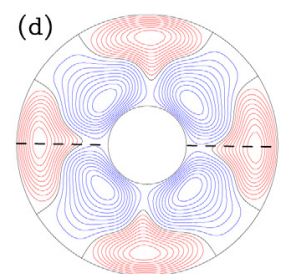

(h)
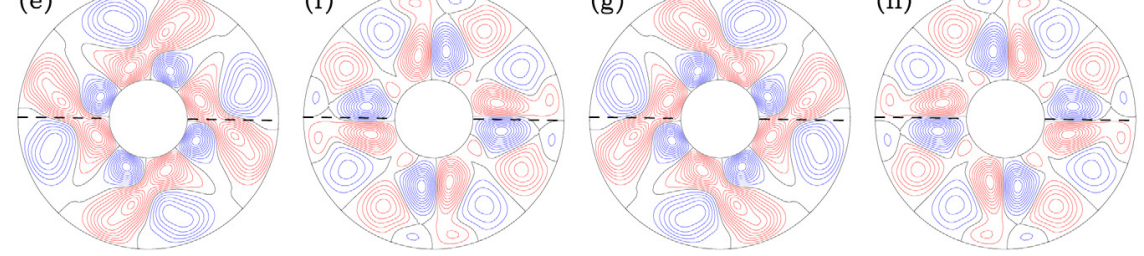

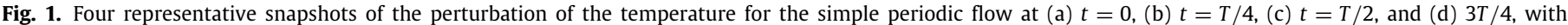
the exact symmetry diameter (as computed below, see Eq. (3.19)) indicated with thick dashed line. (e-h) same snapshots after subtracting the mean field.

be given below with as many significant figures as are estimated to be exact. In particular, the involved frequencies will be computed with seven exact significant figures, which will give a very good computation of the period in periodic dynamics and will help to analyze quasi-periodicity. The involved wavenumbers will be computed even more precisely. We anticipate that these very precise calculations of the frequencies and wavenumbers will be obtained using a limited number of snapshots in a timespan comparable to the period for periodic dynamics and comparable to the largest involved period for quasi-periodic dynamics.

\subsection{Simple periodic flow}

Let us first consider the case of an annulus with radius ratio $\eta=0.3$. The Prantl and Rayleigh numbers are $\operatorname{Pr}=0.025$ (mercury) and $\mathrm{Ra}=10000$, respectively, and the dynamics are periodic with period $T=0.14868194$, which will be computed with these seven significant figures below.

Fig. 1 shows four representative snapshots in the first row, and the same snapshots after subtracting the time average over a period (mean field) in the second. They suggest (but do not ensure) that the pattern exhibits the following instantaneous and spatio-temporal symmetries

$$
\begin{aligned}
& \Theta(r, \phi, t)=\Theta(r, \phi+\pi / 2, t) \text { and } \\
& \Theta\left(r, \phi_{0}+\phi, t\right)=\Theta\left(r, \phi_{0}-\phi, t+T / 2\right),
\end{aligned}
$$

respectively, where the phase $\phi_{0}$ (which, because of the instantaneous symmetry, is defined modulo $\pi / 4$ ), will be precisely computed below. Note that the snapshots in the first row of Fig. 1 are very close to each other, which suggests that the oscillation is almost standing, namely the temperature field approximately just goes up and down; this will be also explained below. The differences between the snapshots become clear after removing the mean field, and the spatio-temporal symmetry becomes obvious. The up-down oscillation is illustrated in Fig. 2, which shows the evolution during four periods of $\Theta\left(r, \phi_{0}-\phi_{1}, t\right)$ and $\Theta\left(r, \phi_{0}+\phi_{1}, t\right)$, where $r=1.246, \phi_{1}=\pi / 5$, and the phase $\phi_{0}$ as defined above. Note that the amplitude of the oscillation is small compared to the mean value and also that the two curves are $T / 2$ out of phase, consistently with the spatio-temporal symmetry of the solution. Also, the oscillation is almost monochromatic, which will also be explained below.

Now, we apply the SKTD method considering only 20 equispaced snapshots in the temporal interval $0 \leq t \leq 0.1412$ (which is slightly smaller than one period), with each snapshot discretized using $I=33$ values of $r$ in the interval $r_{i} \leq r \leq r_{0}$

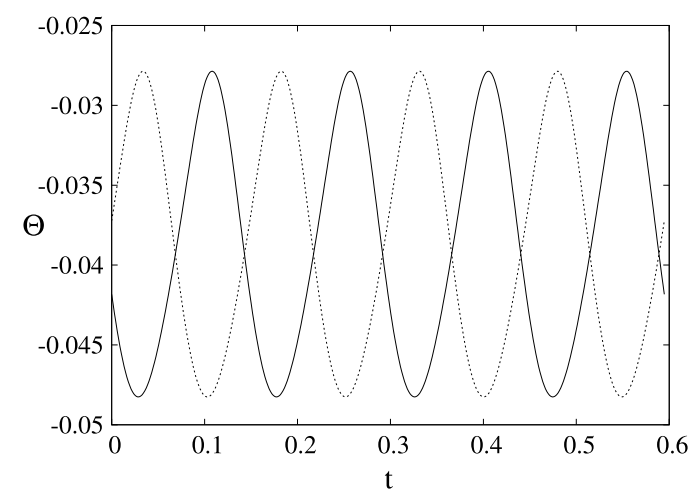

Fig. 2. Time evolution of the temperature at two points symmetrically located around the thick dashed line in Fig. 1.

and $J=51$ equispaced values of $\phi$ in the interval $0 \leq \phi \leq 2 \pi$. Concerning the tunable parameters, we set $\varepsilon_{1}=10^{-8}, \varepsilon_{2}=$ $10^{-3}$, and $d=1$ (although this parameter may be taken in a wide interval, namely $1 \leq d \leq 30$, for both spatial and temporal decompositions, obtaining almost identical results); this flexibility illustrates the robustness of the method, which will also be encountered in the remaining cases considered below. With these values of the tunable parameters, the method retains $2 M+1=11$ spatial modes and $2 N+1=7$ temporal modes. However, instead of the 77 spatio-temporal modes that would in principle appear in the expansion (3.13), condition (1.8) decreases this number to 49 . Retaining these 49 modes, the RRMS error of the spatio-temporal expansion (3.13) (as defined in Eq. (1.9)) is $\sim 2 \times 10^{-3}$. As already explained, the RRMS error would be decreased by decreasing $\varepsilon_{2}$, which would increase the number of retained modes.

The $\kappa-\omega$ diagram associated with the spatio-temporal expansion (3.13) is given in Fig. 3, where only those $(\kappa, \omega)$ pairs associated with nonzero mode amplitudes are displayed. According to this diagram, the expansion (3.13) only contains integer values (with 13 significant figures) of the wavenumber (including the value $\kappa_{0}=0$ ), as expected due to the periodicity in $\phi$. They are all multiples of 4 (with 6 significant figures), which confirms the instantaneous four-fold spatial symmetry in (3.17). The frequencies are all multiples (with six significant figures) of the primary frequency $\omega_{1}=42.259238$, which gives the period of the orbit, $T=2 \pi / \omega_{1}=0.14868194$ that was anticipated above. The spatial and temporal growth rates are $\sim 10^{-13}$ and $\sim 10^{-6}$, 


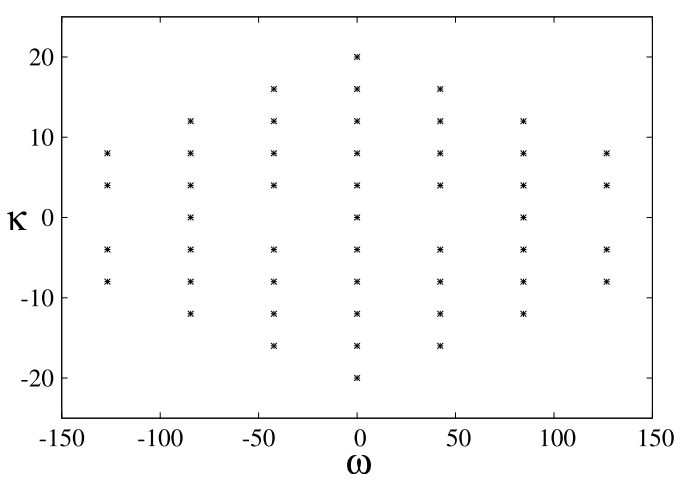

Fig. 3. The $\kappa-\omega$ diagram for the simple periodic flow.

respectively. Thus, the expansion (3.13) can be approximated for the present case as

$\Theta(r, \phi, t)=\sum_{m=-M}^{M} \sum_{n=-N}^{N} a_{m n} u_{m n}(r) \mathrm{e}^{\mathrm{i}\left(4 m \phi+n \omega_{1} t\right)}$,

where, as explained above, some of the amplitudes $a_{m n}=0$. We insist that this expansion is defined for continuous values of $\phi$ and $t$, in spite of the fact that it has been calculated using discrete data in these variables. Also, it is to be noted that retaining only those modes with $m=0$ and $n=0$, we obtain the spatial and temporal thermal mean fields, respectively.

The $a-\kappa$ and $a-\omega$ diagrams associated with the spatial and temporal expansions (3.10) and (3.11) are given in Fig. 4, where it can be seen that the amplitudes in these expansions decay exponentially. Also, the amplitude of the temporal mean field $(\omega=0)$ is much larger than those of the oscillatory modes $(\omega \neq 0)$, which explains the small amplitude of the oscillation, compared to the mean value in Fig. 2. Finally, the amplitudes of the leading nonzero wavenumbers and frequencies are about ten times larger than those for the remaining modes, which explains why the oscillation was almost monochromatic in Fig. 2.

Let us now use the expansion (3.18) to analyze the spatiotemporal symmetries that are present. This is done by checking that the modes exhibiting opposite wave numbers for a fixed frequency are such that

$u_{m n}=\mathrm{e}^{-8 \mathrm{i} m \phi_{0}} u_{-m, n}$ if $n$ even and $u_{m n}=-\mathrm{e}^{-8 \mathrm{i} m \phi_{0}} u_{-m, n}$ if $n$ odd,

where $\phi_{0}=-0.0117170 \mathrm{rad}$. Note that $\phi_{0}$ (which is indicated in Fig. 1) is defined modulo $\pi / 4$, which makes the derivation of (3.19) somewhat tricky. In fact, Eq. (3.19) only permits computing $\phi_{0}$ modulo $\pi /(4 m)$. Here, we have calculated $\phi_{0}$ using the (complex) cosine of the angle formed by the complex modes for $m=$ $\pm 1, n=0$, which equals one with ten significant figures, meaning that $\phi_{0}$ is exact with five significant figures, as anticipated above. Then, we have checked that (3.19) holds with this value of $\phi_{0}$ for the remaining values of $m$ and $n$, also with five significant figures. Eq. (3.19) means that within the approximation relevant here, the expansion (3.18) can be decomposed into two parts, as

$\Theta(r, \phi, t)=\Theta^{\text {even }}(r, \phi, t)+\Theta^{\text {odd }}(r, \phi, t)$,

where

$\Theta^{\text {even }}(r, \phi, t)=\sum_{m=0}^{M} \sum_{\substack{n=-N \\ n \text { even }}}^{N} a_{m n} \tilde{u}_{m n}(r) \mathrm{e}^{\mathrm{i} n \omega_{1} t} \cos \left[4 m\left(\phi-\phi_{0}\right)\right]$,

$$
\Theta^{\text {odd }}(r, \phi, t)=\mathrm{i} \sum_{m=0}^{M} \sum_{\substack{n=-N \\ n \text { odd }}}^{N} a_{m n} \tilde{u}_{m n}(r) \mathrm{e}^{\mathrm{i} n \omega_{1} t} \sin \left[4 m\left(\phi-\phi_{0}\right)\right],
$$

with

$\tilde{u}_{0 n}=u_{0 n}$ and $\tilde{u}_{m n}=2 \mathrm{e}^{4 \mathrm{i} m \phi_{0}} u_{m n}$ if $m>0$.

Thus, $\Theta^{\text {even }}$ and $\Theta^{\text {odd }}$ are symmetric and antisymmetric around $\phi=\phi_{0}$, respectively. Moreover, Eqs. (3.21) and (3.22) imply that both $\Theta^{\text {even }}$ and $\Theta^{\text {odd }}$ satisfy the spatio-temporal symmetry in (3.17), which invoking (3.20) implies that $\Theta$ also exhibits this symmetry. In addition, it has been checked that $\Theta^{\text {even }}$ is about ten times larger than $\Theta^{\text {odd }}$, which means that the whole pattern is a modulated standing wave pattern that slightly oscillates left and right around the diameter $\phi=\phi_{0}, \phi_{0}+\pi$. This explains why the snapshots in Fig. 1 were very close to each other. Similar patterns with a larger amplitude were found in [22] in a short reactiondiffusion system. This type of oscillation can also be interpreted as a wave slightly moving back and forth around the symmetry axis (see [23]).

Summarizing, the SKTD method gave the precise semianalytic expression (3.18) for the considered periodic solution, which moreover, has been decomposed into its symmetric and antisymmetric parts around a symmetry axis that has been computed very precisely. Decomposing the solution into its symmetric and antisymmetric parts around this axis, as in (3.20)-(3.23), has permitted uncovering the instantaneous and spatio-temporal symmetries in (3.17).

\subsection{More complex periodic flow}

As a second application of the HODMD and STKD methods, we consider the case with $\eta=0.3, \operatorname{Pr}=0.025$, and $\mathrm{Ra}=10600$, in which the dynamics are also periodic with period $T=0.1452264$, which will be accurately computed below. Fig. 5 shows four representative snapshots in the first row, and the same snapshots after subtracting the mean field in the second. They suggest that the pattern exhibits again the spatio-temporal symmetry

$\Theta\left(r, \phi_{0}+\phi, t\right)=\Theta\left(r, \phi_{0}-\phi, t+T / 2\right)$,

with $\phi_{0}$ as defined in Eq. (3.26). A careful look at the plots in Fig. 5 shows that the instantaneous four-fold spatial symmetry appearing in Eq. (3.17) approximately holds, but has been broken, which will be confirmed below. As in the case considered in the last subsection, the four initial snapshots in the first row of Fig. 5 are very close to each other, which once more suggest that the whole pattern is approximately (but not exactly, as seen comparing the plots of the second row after removing the mean field) a standing wave pattern, in which the temperature field approximately just moves up and down; this will be explained below. The up-down oscillation is illustrated in Fig. 6, which shows the time evolution during four periods of $\Theta\left(r, \phi_{0}-\phi_{1}, t\right)$ and $\Theta\left(r, \phi_{0}+\phi_{1}, t\right)$, with $r=1.246, \phi_{1}=\pi / 5$, and the phase $\phi_{0}$ is as indicated above (and computed in Eq. (3.26)). Again, the curves are $T / 2$ out of phase, which is consistent with the spatiotemporal symmetry (3.24). Note that the oscillation in Fig. 6 is more complex (namely, less monochromatic) than its counterpart in Fig. 2, which is due to the larger number of modes involved, see Figs. 7 and 8.

Now, we apply the SKTD method considering 34 equispaced snapshots in the temporal interval $0 \leq t \leq 0.2396$, somewhat smaller than two periods, with each snapshot discretized using $I=33$ values of $r$ in the interval $r_{i} \leq r \leq r_{o}$ and $J=101$ equispaced values of $\phi$ in the interval $0 \leq \bar{\phi} \leq 2 \pi$. Note that the number of snapshots and the number of values of $\phi$ are 

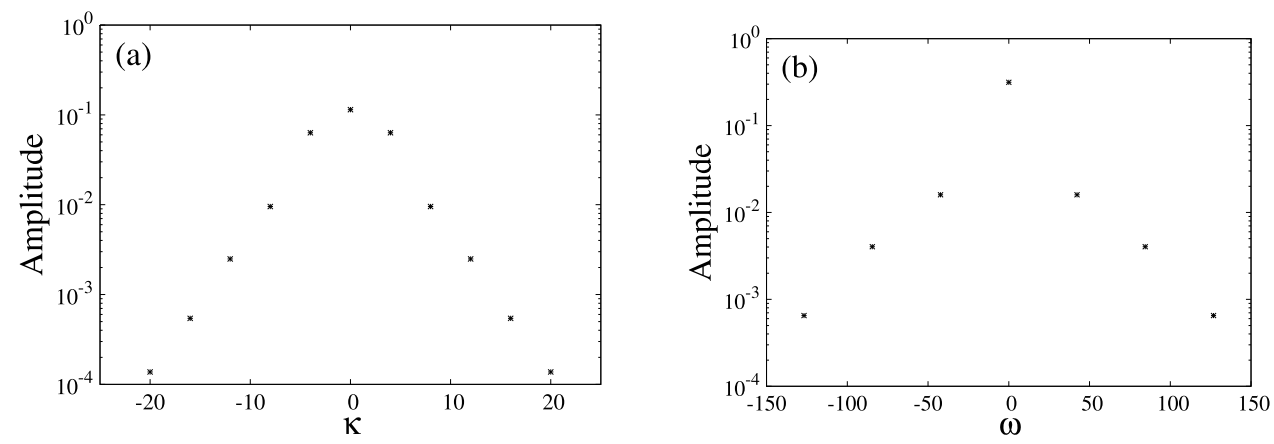

Fig. 4. The (a) $a-\kappa$, and (b) $a-\omega$ diagrams for the simple periodic flow.

(a)

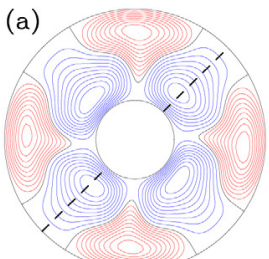

(e) (b)

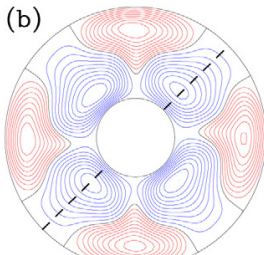

(f)

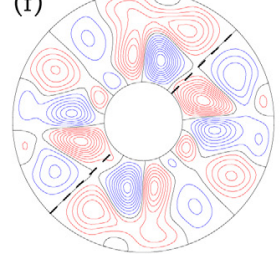

(c)

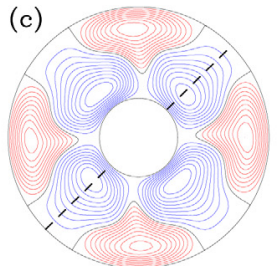

(g)

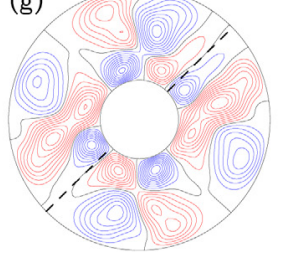

(d)

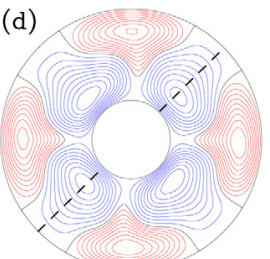

(h)

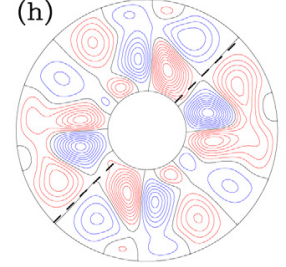

Fig. 5. Counterpart of Fig. 1 for the more complex periodic flow.

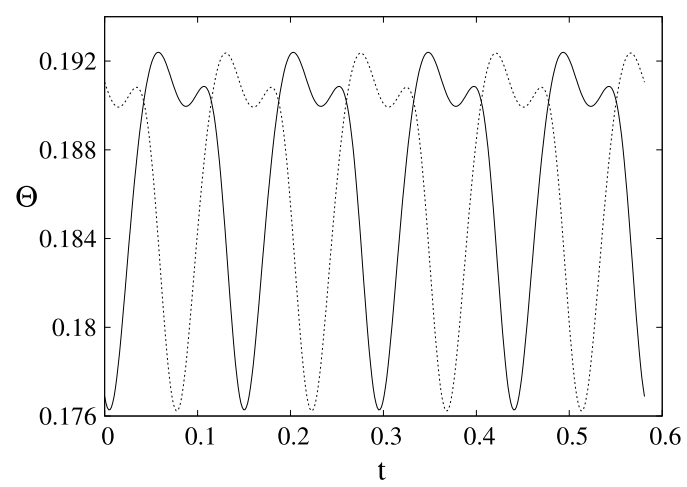

Fig. 6. Counterpart of Fig. 2 for the more complex periodic flow.

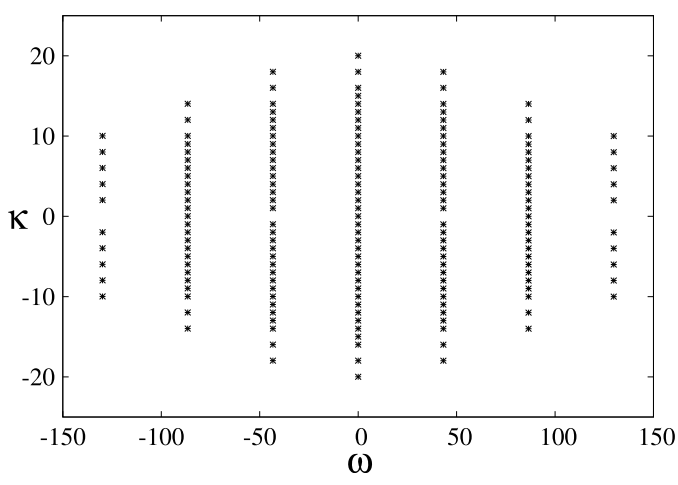

Fig. 7. The $\kappa-\omega$ diagram for the more complex period flow. both larger than in the case considered in the last subsection, which is due to the larger complexity of the flow in the azimuthal direction. Concerning the tunable parameters, we set $\varepsilon_{1}=10^{-8}$, $\varepsilon_{2}=5 \times 10^{-4}$ (smaller than in the former case to obtain a comparable error), and $d=1$ (although, as in the former case, almost identical results are obtained taking this parameter in a wide interval for both space and time, namely $1 \leq d \leq 5$ for both the spatial and temporal decompositions). With these tunable parameters, the method retains $2 M+1=49$ spatial modes and $2 N+1=9$ temporal modes. However, instead of the 441 spatio-temporal modes that would in principle appear in the expansion (3.13), condition (1.8) decreases this number to 171. Retaining these 171 modes, the RRMS error of the spatiotemporal expansion (3.13) (as defined in Eq. (1.9)) is $\sim 2.5 \times$ $10^{-3}$.

The $\kappa-\omega$ diagram associated with the spatio-temporal expansion (3.13) is given in Fig. 7. This figure only exhibits integer values (with 13 significant figures) of the wavenumber, including the values $\kappa_{0}=0$ and $\kappa= \pm 1$. This both explains that the number of involved wavenumbers is larger than in the case considered in the last subsection and means that the instantaneous four-fold symmetry appearing in (3.17) does not strictly apply in the present case. However, it has been checked that those modes with $\kappa$ a positive or negative multiple of 4 exhibit much larger amplitudes (approximately, five times larger, see Fig. 8) than those for the remaining modes. This explains why the four-fold instantaneous symmetry is still approximately seen in Fig. 5 and means that the Rayleigh number for the present case is somewhat close to its value at the bifurcation where this symmetry is broken. Concerning the relevant frequencies in Fig. 7, they are all multiples (with six significant figures) of the primary frequency $\omega_{1}=43.26476$. Thus, the period of the orbit is $T=2 \pi / \omega_{1}=$ 0.1452264 , as indicated above. The spatial and temporal growth 

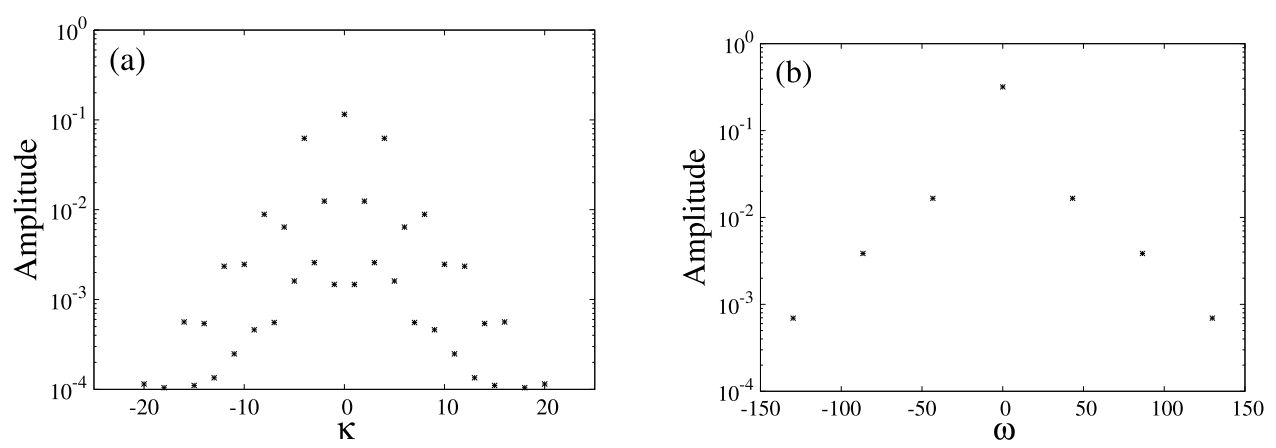

Fig. 8. The (a) $a-\kappa$ and (b) $a-\omega$ diagrams for the more complex period flow.
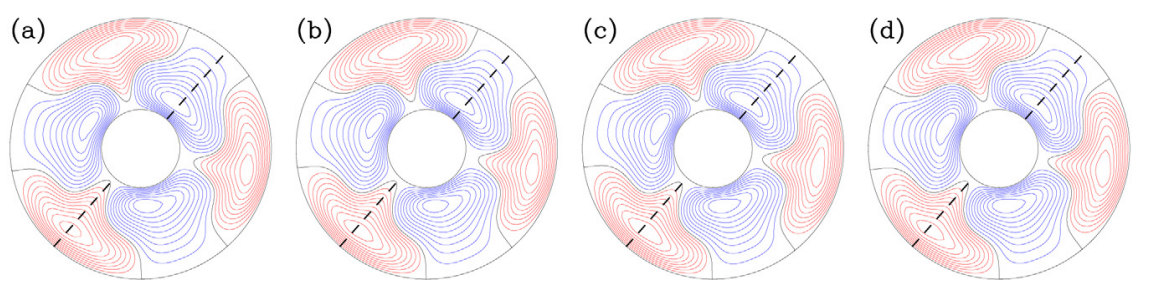

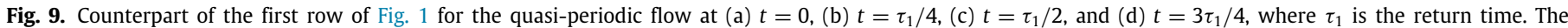
meaning of the axis plotted with thick dashed line will be explained below.

rates are $\sim 10^{-13}$ and $\sim 10^{-6}$, respectively. Thus, the expansion (3.13) can be approximated for the present case as

$\Theta(r, \phi, t)=\sum_{m=-M}^{M} \sum_{n=-N}^{N} a_{m n} u_{m n}(r) \mathrm{e}^{\mathrm{i}\left(m \phi+n \omega_{1} t\right)}$,

where, as in the previous case, some of the amplitudes are $a_{m n}=$ 0 , and this expansion is defined for continuous values of $\phi$ and $t$, in spite of the fact that it has been calculated using discrete data in these variables. Also, it is to be noted that retaining only those modes with $m=0$ and $n=0$, we obtain the spatial and temporal mean fields, respectively.

The $a-\kappa$ and $a-\omega$ diagrams for the spatial and temporal DMD expansions (3.10) and (3.11) are given in Fig. 8. As in the case considered in the last subsection, this figure shows that the mode amplitudes decay exponentially.

The expansion (3.25) can be used to analyze the spatiotemporal symmetries. The counterpart of Eq. (3.19) is

$u_{m n}=\mathrm{e}^{-2 \mathrm{i} m \phi_{0}} u_{-m, n}$ if $n$ even and $u_{m n}=-\mathrm{e}^{-2 \mathrm{i} m \phi_{0}} u_{-m n}$ if $n$ odd,

where $\phi_{0}=0.7780 \mathrm{rad}$. Again, $\phi_{0}$ is computed for the case $m=1$, $n=0$. Then, it is checked that Eq. (3.26) holds with this $\phi_{0}$ for the remaining values of $m$ and $n$. And, once more, Eq. (3.26) means that the expansion (3.25) can be decomposed into two parts, as in Eq. (3.20), with $\Theta^{\text {even }}$ and $\Theta^{\text {odd }}$ given by

$\Theta^{\text {even }}(r, \phi, t)=\sum_{m=0}^{M} \sum_{\substack{n=-N \\ n \text { even }}}^{N} a_{m n} \tilde{u}_{m n}(r) \mathrm{e}^{\mathrm{i} n \omega_{1} t} \cos \left[m\left(\phi-\phi_{0}\right)\right]$,
$\Theta^{\text {odd }}(r, \phi, t)=\mathrm{i} \sum_{m=0}^{M} \sum_{\substack{n=-N \\ n \text { odd }}}^{N} a_{m n} \tilde{u}_{m n}(r) \mathrm{e}^{\mathrm{i} n \omega_{1} t} \sin \left[m\left(\phi-\phi_{0}\right)\right]$,

where

$\tilde{u}_{0 n}=u_{0 n}$ and $\tilde{u}_{m n}=2 \mathrm{e}^{\mathrm{i} m \phi_{0}} u_{m n}$ if $m>0$.

These are the counterparts of (3.21)-(3.23) noting that the present pattern does not exhibit the four-fold symmetry. As in the last subsection, Eqs. (3.27) and (3.28) imply that both $\Theta^{\text {even }}$ and $\Theta^{\text {odd }}$ are invariant under the reflection symmetry (3.24), which in turn implies that $\Theta$ is also invariant under this symmetry. In addition, it has been checked that $\Theta^{\text {even }}$ is about ten times larger than $\Theta^{\text {odd }}$, which means that the whole pattern is again a modulated standing wave pattern that slightly oscillates left and right around the diameter $\phi=\phi_{0}, \phi_{0}+\pi$. This explains why the four snapshots in Fig. 5 were very close to each other.

Summarizing, as for the simpler periodic solution considered in the former subsection, we have obtained a semi-analytic description of the solution and have precisely computed the symmetry axis for the relevant spatio-temporal symmetry. Also, we have explained that the four-fold instantaneous symmetry does not exactly hold in the present case, although it is approximately present.

\subsection{A quasi-periodic flow}

Let us consider now the case with $\eta=0.3 \operatorname{Pr}=0.025$, and $\mathrm{Ra}=11500$, in which the dynamics are seemingly quasiperiodic. In fact, quasi-periodicity is a subtle matter that cannot be ascertained with finite precision computations. Instead, we shall use the high precision in computing the relevant frequencies via the STKD method to address this question. The counterpart of the first row of Figs. 1 and 5 for the present case is given in Fig. 9, where the period $T$ has been substituted by the return time $\tau_{1}$, defined as the first value of $t$ at which the trajectory in phase space conveniently approaches the initial condition; see below. The four considered snapshots, which are representative of the dynamics, show that (i) the flow is conformed by an approximate (but not exact) three fold pattern and (ii) the four snapshots are fairly close to each other, which suggests that the whole pattern represents a slight (quasi-periodic) approximately standing oscillation around the axis defining the global symmetry of the torus that contains the trajectory; see below. The up-down oscillation, illustrated in Fig. 10, shows the evolution during four time units of $\Theta\left(r, \phi_{0}-\phi_{1}, t\right)$ and $\Theta\left(r, \phi_{0}+\phi_{1}, t\right)$, with $r=1.246$, $\phi_{1}=\pi / 5$, and $\phi_{0}$ is the angle formed by the symmetry line in Fig. 9 and the horizontal axis. Note that the oscillation is slowly 


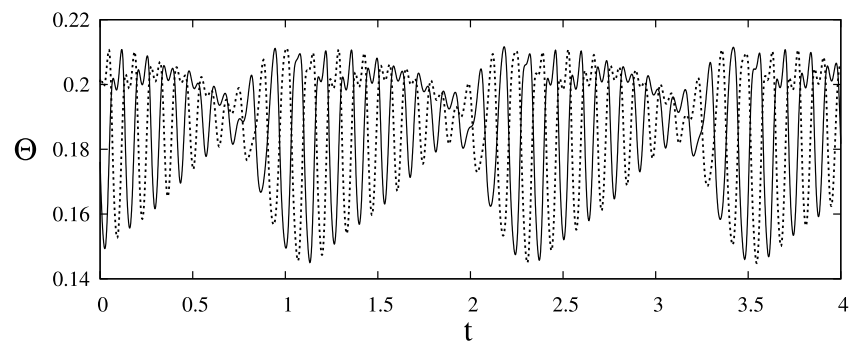

Fig. 10. Counterpart of Fig. 2 for the quasi-periodic flow.
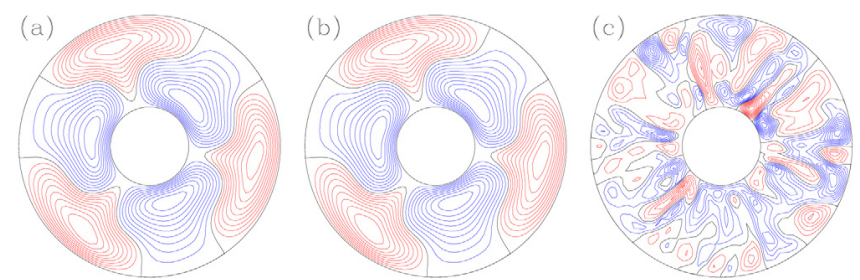

Fig. 11. Contour plots of the temperature for, (a) the left snapshot Fig. 9, (b) its reconstruction using expansion (3.30), and (c) the difference between both.

modulated in time, and that there is an approximate time shift in the oscillation at the two symmetric points.

Now, we apply the SKTD method considering 667 equispaced snapshots in the temporal interval $0 \leq t \leq 4.655$, which is about 3.8 times $t_{2}=2 \pi / \omega_{2}$, (see below for the value of $\omega_{2}$ ). Note that both the considered timespan and the number of snapshots are much larger than in the cases considered in the former subsections, which is due to the more complex quasi-periodic dynamics in the present case. Each snapshot is discretized using $I=33$ values of $r$ in the interval $r_{i} \leq r \leq r_{0}$ and $J=101$ equispaced values of $\phi$ in the interval $0 \leq \phi \leq 2 \pi$. Note that $I$ and $J$ coincide with their counterparts in the last subsection because the present pattern exhibits a similar complexity in the radial and azimuthal directions. Concerning the tunable parameters, we set $\varepsilon_{1}=10^{-8}, \varepsilon_{2}=2 \times 10^{-4}$ (smaller than in the former case to obtain a comparable error), and $d=1$ (although, as in the former cases, this parameter may be taken in a wide interval, namely $1 \leq d \leq 30$ for both the spatial and temporal decompositions to obtain almost identical results). With these values of the tunable parameters, the method retains $2 M+1=61$ spatial modes and $2 N+1=97$ temporal modes. However, instead of the $(2 M+1) \times(2 N+1)=5917$ spatio-temporal modes that would in principle appear in the expansion (3.13), condition (1.8) decreases this number to 1073. Retaining these 1073 spatio-temporal modes, the RRMS error of the spatio-temporal expansion (3.13) (as defined in Eq. (1.9)) is $\sim 3 \times 10^{-3}$. This very small reconstruction error is illustrated in Fig. 11, which shows in this more complex (quasi-periodic) case, the first snapshot in Fig. 9, its reconstruction using the expansion (3.30), and the difference between both. The snapshots lie in the interval $[-4.28,3.08]$ and the difference between both, in the interval $\left[-1.85 \times 10^{-3}, 2.72 \times 10^{-3}\right]$. Thus, the plots Fig. 11(a) and (b) cannot be distinguished.

The $a-\kappa$ and $a-\omega$ diagrams, associated with the spatial and temporal expansions (3.10) and (3.11) are given in Fig. 12, which shows the exponential decay of the spatial and temporal mode amplitudes. The involved values of the wavenumber are all integer numbers (with 13 significant figures). Also, as in the periodic dynamics considered in the former subsection, the minimum positive wavenumber is 1 , which explains that the approximate three-fold instantaneous symmetry that is seen in Fig. 9 is not exact. However, according to Fig. 12(a), the mode amplitudes of
Table 1

Frequencies, integer factors $n_{1}$ and $n_{2}$, and relative error $\left|\omega-n_{1} \omega_{1}-n_{2} \omega_{2}\right| /|\omega|$ with $\omega_{1}=45.59202$ and $\omega_{2}=5.184255$.

\begin{tabular}{llllllll}
\hline $10^{-3} \omega$ & Error & $n_{1}$ & $n_{2}$ & $10^{-2} \omega$ & Error & $n_{1}$ & $n_{2}$ \\
\hline 0.187553 & $0.18 \times 10^{-5}$ & 4 & 1 & 0.963683 & $0.23 \times 10^{-6}$ & 2 & 1 \\
0.182368 & $0.10 \times 10^{-5}$ & 4 & 0 & 0.911840 & $0.16 \times 10^{-6}$ & 2 & 0 \\
0.177184 & $0.30 \times 10^{-5}$ & 4 & -1 & 0.859998 & $0.28 \times 10^{-6}$ & 2 & -1 \\
0.152331 & $0.17 \times 10^{-4}$ & 3 & 3 & 0.808157 & $0.24 \times 10^{-5}$ & 2 & -2 \\
0.147144 & $0.27 \times 10^{-5}$ & 3 & 2 & 0.663300 & $0.15 \times 10^{-4}$ & 1 & 4 \\
0.141960 & $0.98 \times 10^{-6}$ & 3 & 1 & 0.611444 & $0.70 \times 10^{-5}$ & 1 & 3 \\
0.136776 & $0.18 \times 10^{-6}$ & 3 & 0 & 0.559605 & $0.12 \times 10^{-5}$ & 1 & 2 \\
0.131593 & $0.45 \times 10^{-6}$ & 3 & -1 & 0.507763 & $0.81 \times 10^{-6}$ & 1 & 1 \\
0.126407 & $0.91 \times 10^{-6}$ & 3 & -2 & 0.404077 & $0.97 \times 10^{-6}$ & 1 & -1 \\
0.111910 & $0.96 \times 10^{-4}$ & 2 & 4 & 0.352233 & $0.53 \times 10^{-5}$ & 1 & -2 \\
0.106736 & $0.89 \times 10^{-5}$ & 2 & 3 & 0.155523 & $0.28 \times 10^{-4}$ & 0 & 3 \\
0.101553 & $0.26 \times 10^{-5}$ & 2 & 2 & 0.103686 & $0.46 \times 10^{-5}$ & 0 & 2 \\
\hline
\end{tabular}

those modes associated with wavenumbers that are positive or negative multiples of three are much larger than the remaining ones, which explains the approximate three-fold symmetry. Note that the $a-\omega$ diagram is organized in an overall triangular pattern (indicated with thin solid lines in Fig. 12), which is typical of quasi-periodic dynamics [8]. Moreover, the overall triangular pattern is formed by smaller sub-triangles, also indicated in Fig. 12. Each of these sub-triangles gives a slowly modulated pattern with a monochromatic carrier, whose frequency is the vertex of the sub-triangle. The whole pattern is the superposition of these patterns and thus, it is more complex. The upper vertices of the sub-triangles in Fig. 12(b) are located at $\omega=0, \pm \omega_{1}, \pm 2 \omega_{1}, \ldots$, where $\omega_{1}=45.59202$ is computed with seven exact significant figures. On the other hand, the smallest positive frequency appearing in this diagram, $\omega_{2}=5.184255$, is also computed with seven exact significant figures. The frequency $\omega_{1}$ is much larger than $\omega_{2}$, which is very clearly illustrated in the $\kappa-\omega$ diagram associated with the spatio-temporal expansion (3.13), plotted in Fig. 13. The much larger value of $\omega_{1}$ compared to $\omega_{2}$ explains why the oscillation shown in Fig. 10 is a slowly modulated wave, of carrier frequency $\omega_{1}$ and modulating frequency $\omega_{2}$. Note that the $(\omega, \kappa)$ pairs in Fig. 13 accumulate in clusters, which in fact are the counterparts in this figure of the sub-triangles in Fig. 12(b).

The frequencies $\omega_{1}$ and $\omega_{2}$ can be seen as the fundamental frequencies of the quasi-periodic pattern. This is because the remaining frequencies appearing in the expansion (3.13), are all (very approximately) linear combinations with integer coefficients of the primary frequencies, of $\omega_{1}$ and $\omega_{2}$. This can be seen in Table 1 . Note that these values of $n_{1}$ and $n_{2}$ are consistent with what is seen in the $\kappa-\omega$ diagram in Fig. 13. As already explained, in finite-precision computations, it is not possible to decide whether $\omega_{1}$ and $\omega_{2}$ are incommensurable or not. However, in the appendix we show that $\omega_{2} / \omega_{1}=0.1137097 \simeq 141 / 1240$ with a relative error $\sim 0.3 \times 10^{-7}$. Therefore in case of being in resonance, this is very weak.

The relevant frequencies (and thus $\omega_{1}, \omega_{2}$, and the ratio $r=$ $\left.\omega_{2} / \omega_{1}\right)$ could also be obtained from a FFT of the time evolution of a scalar quantity, for instance, the value of $\Theta$ at a given radius and angle. This requires a very long integration time to have enough precision. The SKTD method, instead, provides the same resolution for a shorter time interval of about $1.5 t_{2}$ with $t_{2}=2 \pi / \omega_{2}$, and uses all values of $\Theta$ contained in the snapshots, ensuring that these values of $\omega_{1}$ and $\omega_{2}$ are appropriate for the whole spatial domain.

On the other hand, the spatial and temporal growth rates are $\sim 10^{-13}$ and $\sim 10^{-3}$, respectively. Thus, the expansion (3.13) can be approximated for the present case as

$\Theta(r, \phi, t)=\sum_{m=-M}^{M} \sum_{n_{1}, n_{2}} a_{m n_{1} n_{2}} u_{m n_{1} n_{2}}(r) \mathrm{e}^{\mathrm{i}\left[m \phi+\left(n_{1} \omega_{1}+n_{2} \omega_{2}\right) t\right]}$, 

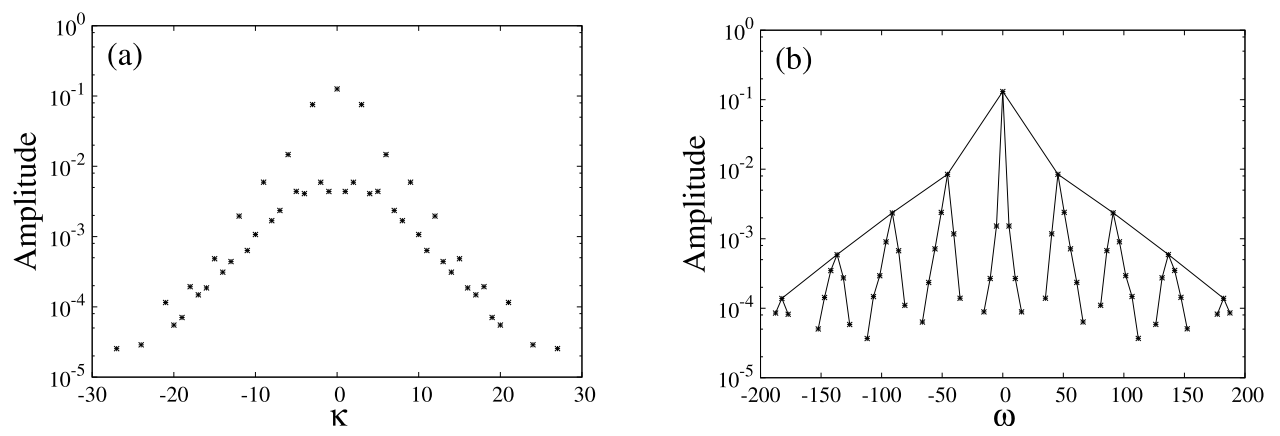

Fig. 12. The (a) $a-\kappa$ and (b) $a-\omega$ diagrams for the quasi-periodic flow.

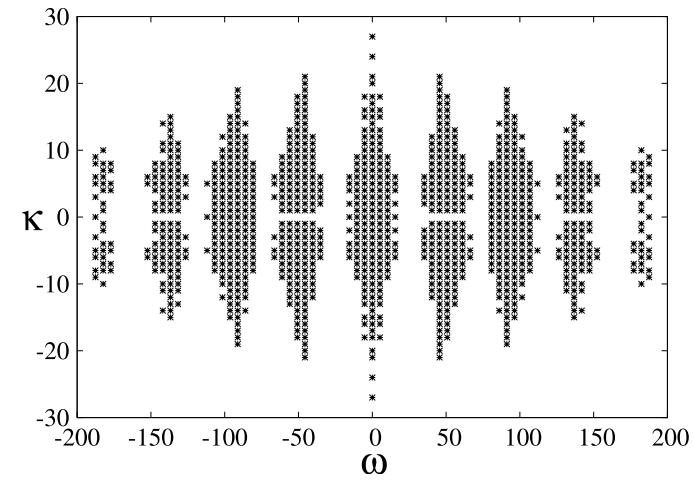

Fig. 13. The $\kappa-\omega$ diagram for the quasi-periodic flow.

where, as in the previous cases, some of the amplitudes are $a_{m n_{1} n_{2}}=0$, and this expansion is defined for continuous values of $\phi$ and $t$, in spite of the fact that it has been calculated using discrete data in these variables. Note that although $10^{-3}$ can seem as non-negligible, the temporal growth rates only achieve this value for the smaller amplitudes. We have checked that neglecting $\delta_{n}$ only introduces changes of $\sim 10^{-7}$ in the spatiotemporal expansion, which is smaller than the relevant accuracy of this expansion. Also, it is to be noted that retaining only those modes with $m=0$ and $n=0$, we obtain the spatial and temporal mean fields, respectively.

The expansion (3.30) permits computing the return time $\tau_{1}$ appearing in Fig. 9, as that value of $t$ for which the trajectory in phase space approaches the initial condition. To this end, we need that $\exp \left(\mathrm{i}\left(n_{1} \omega_{1}+n_{2} \omega_{2}\right) t\right)$ be close to 1 simultaneously for all $n_{1}$ and $n_{2}$. Let $r=\omega_{2} / \omega_{1}$ and $p / q=141 / 1240$ be the rational approximation (with a relative error $\sim 0.3 \times 10^{-7}$ ) of $r$ computed in the appendix. If $\tau_{1}=2 q \pi / \omega_{1}$ (or any integer multiple of this value), then $\exp \left(\mathrm{i}\left(n_{1} \omega_{1}+n_{2} \omega_{2}\right) \tau_{1}\right) \simeq \exp \left(2 \mathrm{i}\left(q n_{1}+p n_{2}\right) \pi\right)=1$ with the relative error anticipated above, as required.

Note that, if the approximating fraction is $p / q=141 / 1240$ then $\tau_{1}$ involves 1240 oscillations with the largest fundamental frequency, or 141 oscillations with the smallest frequency, a time span that is much larger than that covered by the snapshots used to compute the STKD approximation. This illustrates the known ability of the HODMD and STKD methods for temporal extrapolation of the dynamics [6-8].

As in the previous cases, the expansion (3.30) can be used to analyze the spatio-temporal symmetries that are present. It turns out that the counterpart of Eq. (3.26) for the present case is

$u_{m n_{1} n_{2}}=\mathrm{e}^{-2 \mathrm{i} m \phi_{0}} u_{-m n_{1} n_{2}}$ if $n_{1}$ even,

and

$u_{m n_{1} n_{2}}=-\mathrm{e}^{-2 \mathrm{i} m \phi_{0}} u_{-m n_{1} n_{2}}$ if $n_{1}$ odd, where $\phi_{0}=0.8465290 \mathrm{rad}$. As for the periodic dynamics considered in the previous subsections, $\phi_{0}$ is first computed for the case $m=1, n_{1}=n_{2}=0$ and then checked that Eqs. (3.31) and (3.32) hold with this value of $\phi_{0}$ for the remaining values of $m, n_{1}$, and $n_{2}$. The symmetry above could be expected because when a symmetric cycle undergoes a Neimark-Sacker bifurcation, the emerging tori are, generically, invariant as a set relatively to the symmetry of the cycle [24]; in fact, the invariant torus (and its symmetries) associated with the quasi-periodic dynamics (3.31) will be computed below. It is important to note that the spatio-temporal symmetry given by Eqs. (3.31) and (3.32) depends only on the parity of $n_{1}$, which is associated to the frequency $\omega_{1}$, because it is inherited from that of the periodic orbit from which it bifurcates. As explained above, there are alternative methods [23] to compute $\phi_{0}$ (and the return time $\tau_{1}$ ) with a comparable accuracy, but these methods are based on scalar data and require much larger timespans.

As in the previous subsections, Eqs. (3.31) and (3.32) imply that the expansion (3.30) can be decomposed into its symmetric and antisymmetric parts, as

$\Theta(r, \phi, t)=\Theta^{\text {even }}(r, \phi, t)+\Theta^{\text {odd }}(r, \phi, t)$,

where

$\Theta^{\text {even }}=\sum_{m=0}^{M} \sum_{\substack{n_{1}, n_{2} \\ n_{1} \text { even }}} a_{m n_{1} n_{2}} \tilde{u}_{m n_{1} n_{2}}(r) \mathrm{e}^{\mathrm{i}\left(n_{1} \omega_{1}+n_{2} \omega_{2}\right) t} \cos \left[m\left(\phi-\phi_{0}\right)\right]$,

and

$\Theta^{\text {odd }}=\mathrm{i} \sum_{m=0}^{M} \sum_{\substack{n_{1}, n_{2} \\ n_{1} \text { odd }}} a_{m n_{1} n_{2}} \tilde{u}_{m n_{1} n_{2}}(r) \mathrm{e}^{\mathrm{i}\left(n_{1} \omega_{1}+n_{2} \omega_{2}\right) t} \sin \left[m\left(\phi-\phi_{0}\right)\right]$.

Here,

$\tilde{u}_{0 n_{1} n_{2}}=u_{0 n_{1} n_{2}}$ and $\tilde{u}_{m n_{1} n_{2}}=2 \mathrm{e}^{\mathrm{i} m \phi_{0}} u_{m n_{1} n_{2}}$ if $m>1$.

In addition, it has been checked that $\Theta^{\text {even }}$ is about ten times larger than $\Theta^{\text {odd }}$, which means that the whole pattern is a modulated standing wave pattern that slightly oscillates left and right around the lines $\phi=\phi_{0}$. This explains why the four snapshots in Fig. 9 are very close to each other.

Because of the quasi-periodicity, Eqs. (3.33)-(3.35) do not imply any invariance under spatio-temporal reflection of the particular solution that is being considered. However the invariant torus that is densely covered by the solution does exhibit a reflection symmetry, as is seen now. To this end, we obtain a semi-analytic expression of the torus in terms of two curvilinear coordinates, $\theta_{1}$ and $\theta_{2}$. This is done by just replacing in (3.30) $\omega_{1} t$ 


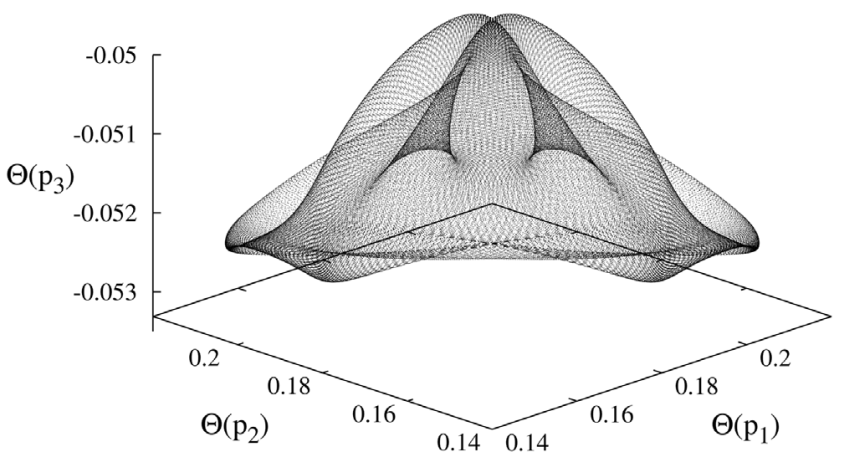

Fig. 14. Three-dimensional projection of the trajectory plotted in Fig. 10. The points $p_{1}, p_{2}$, and $p_{3}$ are located at $(r, \phi)$ with $r=1.246$, and $\phi=\phi_{0}-\pi / 5$, $\phi_{0}+\pi / 5$ and $\phi_{0}$.

and $\omega_{2} t$ by $\theta_{1}$ and $\theta_{2}$, respectively, which yields

$\Theta\left(r, \phi, \theta_{1}, \theta_{2}\right)=\sum_{m=-M}^{M} \sum_{n_{1}, n_{2}} a_{m n_{1} n_{2}} u_{m n_{1} n_{2}}(r) \mathrm{e}^{\mathrm{i}\left(m \phi+n_{1} \theta_{1}+n_{2} \theta_{2}\right)}$.

Obviously, this parametrization of the torus is periodic with period $2 \pi$ in both $\theta_{1}$ and $\theta_{2}$. On the other hand, as we did above with the particular orbit, the parametrization can be decomposed into its symmetric and antisymmetric parts as

$\Theta\left(r, \phi, \theta_{1}, \theta_{2}\right)=\Theta^{\text {even }}\left(r, \phi, \theta_{1}, \theta_{2}\right)+\Theta^{\text {odd }}\left(r, \phi, \theta_{1}, \theta_{2}\right)$,

where

$\Theta^{\text {even }}=\sum_{m=0}^{M} \sum_{\substack{n_{1}, n_{2} \\ n_{1} \text { even }}} a_{m n_{1} n_{2}} \tilde{u}_{m n_{1} n_{2}}(r) \mathrm{e}^{\mathrm{i}\left(n_{1} \theta_{1}+n_{2} \theta_{2}\right)} \cos \left[m\left(\phi-\phi_{0}\right)\right]$,

and

$\Theta^{\text {odd }}=\mathrm{i} \sum_{m=0}^{M} \sum_{\substack{n_{1}, n_{2} \\ n_{1} \text { odd }}} a_{m n_{1} n_{2}} \tilde{u}_{m n_{1} n_{2}}(r) \mathrm{e}^{\mathrm{i}\left(n_{1} \theta_{1}+n_{2} \theta_{2}\right)} \sin \left[m\left(\phi-\phi_{0}\right)\right]$,

with $\tilde{u}_{m n_{1} n_{2}}$ as defined in (3.36). Now, Eqs. (3.39) and (3.40) show that $\Theta^{\text {even }}$ and $\Theta^{\text {odd }}$ are both invariant under the action

$\theta_{1} \rightarrow \theta_{1}+\pi, \quad \phi \rightarrow \phi_{0}-\phi, \quad \Theta \rightarrow \Theta$,

which invoking (3.38) is a reflection symmetry of the whole torus.

Unfortunately, it is not possible to illustrate, neither the semi-analytical description of the whole torus nor the reflection symmetry, in the high-dimensional phase space. Thus, we only consider in Fig. 14 a three-dimensional projection of the torus in the three dimensional space spanned by $\Theta\left(r, \phi_{0}-\phi_{1}\right), \Theta\left(r, \phi_{0}+\right.$ $\left.\phi_{1}\right)$, and $\Theta\left(r, \phi_{0}\right)$, with $r=1.246$, and $\phi_{1}=\pi / 5$. In fact, for the sake of clarity, we have plotted a very long trajectory, instead of the whole torus. Note that in this perspective the symmetry plane is the vertical plane containing the horizontal diagonal. Also note that the projected torus exhibits self-intersections.

Summarizing, as in the previous sub-sections, we have explained how the dynamics correspond to an approximately standing, slowly modulated, seemingly quasi-periodic oscillation that approximately (but not exactly) exhibits a three-fold symmetry. In addition, we have derived semi-analytic descriptions of both the considered particular dynamics and the densely covered torus. Finally, we have shown that the torus is invariant under a reflection symmetry.

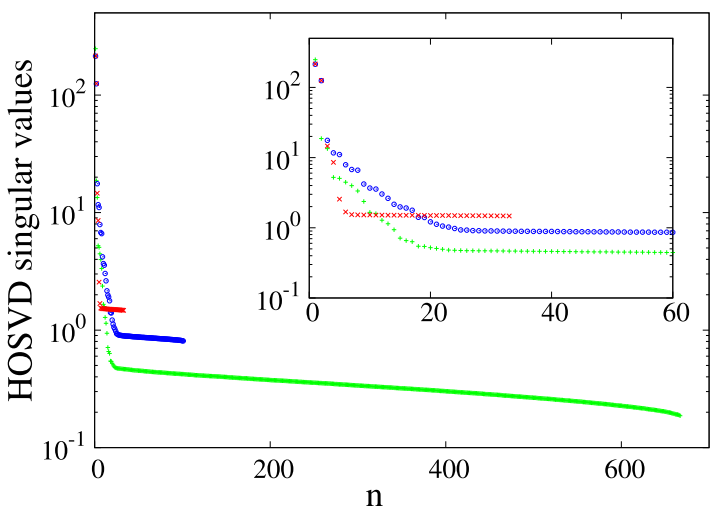

Fig. 15. HOSVD singular values along the radial (red), azimuthal (blue), and temporal (green) directions. (For interpretation of the references to color in this figure legend, the reader is referred to the web version of this article.)

\section{Noisy quasiperiodic flow}

In the former section, the indexes in the application of the spatial and temporal DMD methods involved in the STKD method (see Section 2) were both equal to 1 , namely $d^{x}=d^{t}=1$. This was because the amount of spatio-temporal data was very large and the data was very clean.

Let us add some noise to the quasiperiodic thermal data considered in Section 3.3. To this end, we consider the associated snapshot tensor (depending on three indexes), denoted in this section as the clean tensor, $\Theta^{\text {clean }}\left(r_{i}, \phi_{j}, t_{k}\right)$, for $i=1, \ldots, I, j=$ $1, \ldots, J$, and $t=1, \ldots, K$, with $I=33, J=101$, and $K=667$.

Now, we define a noisy tensor as

$\Theta^{\text {noisy }}=\Theta^{\text {clean }}+$ Noise,

where, using the MATLAB command 'rand', the $I \times J \times K$-noise tensor is defined as

Noise $=0.02[\operatorname{rand}(I, J, K)-0.5]$.

Using these, the relative RMS difference between the clean and noisy tensors turns out to be

RRMS error $\equiv \frac{\left\|\Theta^{\text {noisy }}-\Theta^{\text {clean }}\right\|_{2}}{\left\|\Theta^{\text {clean }}\right\|_{2}}=0.035$,

where $\|\cdot\|_{2}$ is the Frobenius norm.

Let us now apply the truncated HOSVD method to the noisy tensor, which reads

$\Theta\left(\boldsymbol{y}_{i}, x_{j}, t_{k}\right)=\sum_{p_{1}=1}^{P_{1}} \sum_{p_{2}=1}^{P_{2}} \sum_{p_{3}=1}^{P_{3}} S_{p_{1} p_{2} p_{3}} v_{\boldsymbol{y}_{i} p_{1}}^{1} v_{x_{j} p_{2}}^{2} v_{t_{k} p_{3}}^{3}$.

Here, we retain all modes, namely we set $P_{1}=I, P_{2}=J$, and $P_{3}=K$. The resulting HOSVD singular values are plotted in Fig. 15, where it is easily seen a change of tendency in the singular values along the three directions. A careful inspection at the blow up in Fig. 15 shows that the change of tendency occurs at $P_{1}=10$, $P_{2}=26$, and $P_{3}=25$ along the radial, azimuthal, and temporal directions, respectively. Retaining precisely these numbers of modes in (4.4), the resulting truncated tensor is denoted as $\Theta^{\text {trunc. }}$. Now, we apply the STKD method to this truncated tensor using the following thresholds

$\varepsilon_{1}=4 \cdot 10^{-4}$ and $\varepsilon_{2}=10^{-4}$

for dimension reduction and mode truncation, respectively, and the following indexes for the DMD-d along the azimuthal and temporal directions

$d^{\phi}=5$ and $d^{t}=200$ 
respectively. These parameters are chosen after some slight calibration. The resulting reconstructed tensor, denoted as $\Theta^{\text {reconst. }}$ uses 3315 spatio-temporal modes and gives a relative RMS error

RRMS error $\equiv \frac{\left\|\Theta^{\text {reconst. }}-\Theta^{\text {clean }}\right\|_{2}}{\left\|\Theta^{\text {clean }}\right\|_{2}}=0.0055$,

which is more than six times smaller than its counterpart for the noisy tensor (see Eq. (4.3)). Thus, the ability of the STKD method for noise filtration has been illustrated. On the other hand, if standard DMD is used to apply the STKD method in both space and time, namely if the values in (4.6) for the spatial and temporal indexes are replaced by

$d^{\phi}=d^{t}=1$

then the counterpart of (4.7) for the reconstruction turns out to be

RRMS error $\equiv \frac{\left\|\Theta^{\text {reconst. }}-\Theta^{\text {clean }}\right\|_{2}}{\left\|\Theta^{\text {clean }}\right\|_{2}}=0.045$,

which is even larger than its counterpart for the noisy tensor, as comparison with (4.3) shows. In other words, using standard DMD to apply the STKD method does not filter errors in the present case.

\section{Conclusions}

The SKTD has been used to analyze periodic and quasi-periodic solutions obtained by numerical simulation of a discretized set of partial differential equations. It has been shown that it is a very efficient method for this purpose. In the present case, because the amount of data is very large, the combination of the STKD method with standard DMD has given good results when using clean data (as provided by the numerical solver). However, it has been shown that for noisy data, in order to filter noise, the STKD method must be combined with the strictly HODMD method, namely with the algorithm DMD- $d$ with $d>1$.

The first important point is that the method is valid for any type of discretization. Here, the azimuthal direction was discretized with Fourier expansions, but the snapshots used in the analysis were written at a mesh of equally-spaced azimuthal collocation points, then with a mesh of finite elements or finite differences the results would had been the same.

STKD techniques exhibit several advantages compared to other methods of analysis, specially for quasi-periodic dynamics. For instance, they are able to provide accurate dispersion relations of the solution with snapshots in a time interval of about 1.5 times $t_{2}=2 \pi / \omega_{2}$, while good frequency spectra via FFTs requires very long integration times, which for $3 \mathrm{D}$ solutions is always very computationally expensive. One of the reasons is that the FFTs are usually applied to representative scalars of the fields, while SKTD uses information from all spatial points contained in the snapshots simultaneously. For the same reason, if spatial or spatio-temporal symmetries are present, they are obtained as a byproduct, by just looking at the relations between the computed modes. It is possible then to find a splitting of the solutions into their symmetric and antisymmetric parts. This fact facilitates constructing ad hoc simple models to reduce the cost of the study.

In the particular applications considered above, the periodic or quasi-periodic solutions exhibited some exact or approximate instantaneous or spatio-temporal symmetries, which were very precisely identified by the method. Also, for quasi-periodic solutions, the return time was seen to be very large, which ensures that, if present, the underlying resonance is very weak. Note that such resonances (resulting from, e.g., Arnold tongues, [24])
Table 2

Fractions $p / q$ approximating the ratio $\omega_{2} / \omega_{1}$ and the associated errors, $\left|p / q-\omega_{2} / \omega_{1}\right|$.

\begin{tabular}{rrlrrl}
\multicolumn{1}{c}{$p$} & $q$ & $\omega_{2} / \omega_{1}-p / q$ & $p$ & $q$ & $\omega_{2} / \omega_{1}-p / q$ \\
\hline 0 & 1 & $0.114 \times 10^{0}$ & 29 & 255 & $0.158 \times 10^{-4}$ \\
1 & 1 & $0.886 \times 10^{0}$ & 34 & 299 & $0.267 \times 10^{-5}$ \\
1 & 5 & $0.863 \times 10^{-1}$ & 73 & 642 & $0.254 \times 10^{-5}$ \\
1 & 6 & $0.530 \times 10^{-1}$ & 107 & 941 & $0.886 \times 10^{-6}$ \\
1 & 7 & $0.291 \times 10^{-1}$ & 141 & 1240 & $0.287 \times 10^{-7}$ \\
1 & 8 & $0.113 \times 10^{-1}$ & & & \\
1 & 9 & $0.260 \times 10^{-2}$ & 1726 & 15179 & $0.245 \times 10^{-7}$ \\
3 & 26 & $0.167 \times 10^{-2}$ & 1867 & 16419 & $0.205 \times 10^{-7}$ \\
4 & 35 & $0.576 \times 10^{-3}$ & 2008 & 17659 & $0.170 \times 10^{-7}$ \\
5 & 44 & $0.733 \times 10^{-4}$ & 2149 & 18899 & $0.140 \times 10^{-7}$ \\
19 & 167 & $0.627 \times 10^{-4}$ & 2290 & 20139 & $0.114 \times 10^{-7}$ \\
24 & 211 & $0.344 \times 10^{-4}$ & 2431 & 21379 & $0.906 \times 10^{-8}$ \\
\hline
\end{tabular}

cannot be distinguished from genuine quasi-periodic dynamics with finite precision computations.

The expansions obtained by the SKTD are valid for any value of time and any value of the spatial points. Therefore, in the case of quasi-periodic solutions, the same expansion provides not only a semi-analytic approximation of the particular trajectory, which is dense in a torus, but also a similar semi-analytic expression for the full invariant torus, no matter the number of retained independent frequencies. In the case considered in this paper, the quasi-periodic solution was associated with a 2-torus, but the method would obviously be appropriate to deal with higher dimension tori.

\section{Uncited references}

\section{Acknowledgments}

This work has been partially supported by the Spanish Ministry of Economy and Competitiveness and European Regional Development Fund, under grants MINECO/FEDER TRA2016-75075-R and FIS2016-76525-P, and by the Generalitat de Catalunya under grant AGAUR 2017-SGR-1374. We are also indebted to two anonymous referees for several useful comments on an earlier version of the paper, which helped to improve presentation of the results.

\section{Appendix. Approximation of irrational numbers by irreducible fractions}

Given an irrational number $\rho$ (or an approximation of $\rho$ with machine error, also denoted as $\rho$ ), an algorithm to find rational approximations $p / q$ of $\rho$ using Haros-Farey fractions [25] proceeds as follows. Let $b / d$ and $a / c$ be two initial irreducible fractions such that $b / d<\rho<a / c)$. They can be, for instance, $\operatorname{int}(\rho) / 1$ and $\operatorname{int}(\rho)+1) / 1$, where $\operatorname{int}(\rho)$ denotes the integer part of $\rho$. The interval is narrowed at each step by computing the mediant of the two fractions $(a+b) /(c+d)$ (which is also irreducible) and deciding which of the two intervals $[b / d,(a+$ $b) /(c+d)],[(a+b) /(c+d), a / c]$ contains $\rho$. The process is repeated until the distance $|(a+b) /(c+d)-\rho|$ is small enough. By taking at each step the limit of the interval closest to $\rho$, a sequence of irreducible rational number is obtained, which converges to $\rho$ with monotonically decreasing error.

Table 2 shows the sequence of approximations when the algorithm is applied to $\rho=\omega_{2} / \omega_{1}=0.1137097060783770$ (with $\omega_{1}$ and $\omega_{2}$ as given by the STKD method retaining all significant figures, in spite of the fact that only seven significant figures are expected to be exact), with their corresponding error. 
If $p / q=141 / 1240=0.11370968$ the error is $\sim 0.3 \times 10^{-7}$. Note that beyond this error value, the table contains slightly better approximating fractions, but with much larger (about ten times larger) values of $p$ and $q$. This is consistent with our anticipated statement that the values of $\omega_{1}$ and $\omega_{2}$ (and thus the value of $\rho$ ) provided by the STKD method are exact with seven significant figures.

\section{References}

[1] M.M. Zdravkovich, Different modes of vortex shedding: an overview, J. Fluid Mech. 10 (1996) 427-437.

[2] E. Dowell, A Modern Course in Aeroelasticity, Springer, Berlin, 1998.

[3] M. Lappa, Thermal Convection: Patterns Evolution and Stability, Wiley and Sons, Singapore, 2010

[4] M. Lappa, Rotating Thermal Flows in Natural and Industrial Processes, Wiley and Sons, Singapore, 2012.

[5] P.J. Schmid, Dynamic mode decomposition of numerical and experimental data, J. Fluid Mech. 656 (2010) 5-28.

[6] S. Le Clainche, J.M. Vega, Higher order dynamic mode decomposition, SIAM J. Appl. Dyn. Syst. 16 (2017) 882-925.

[7] S. Le Clainche, J.M. Vega, Higher order dynamic mode decomposition to identify and extrapolate flow patterns, Phys. Fluids 29 (2017) 084102

[8] S. Le Clainche, J.M. Vega, Spatio-temporal Koopman decomposition, J. Nonlinear Sci. 28 (2018) 1793-1842.

[9] W.H. Press, S.A. Teukolsky, W.T. Vetterling, B.P. Flannery, Numerical Recipes in C, Cambridge Univ. Press, 1988.

[10] J. Laskar, Frequency analysis of a dynamical system, Celest. Mech. Dyn. Astron. 56 (1) (1993) 191-196.

[11] G. Gómez, J.M. Mondelo, C. Simó, A collocation method for the numerical Fourier analysis of quasi-periodic functions. I: Numerical tests and examples, Discrete Contin. Dyn. Syst. Series B 14 (1) (2010) 41-74.
[12] M. Cross, P. Hohenberg, Pattern formation outside of equilibrium, Rev. Modern Phys. 65 (1993) 851-1112.

[13] J.M. Vega, E. Knobloch, C. Martel, Nearly inviscid Faraday waves in annular containers of moderately large aspect ratio, Physica D 154 (2001) 313-336.

[14] J. Sánchez, M. Net, J.M. Vega, Amplitude equations close to a triple(+1) bifurcation point of $D_{4}$-symmetric periodic orbits in $O(2)$-equivariant systems, Discrete Contin. Dyn. Syst. Series B 6 (6) (2006) 1357-1380.

[15] M. Haragus, G. Iooss, Local Bifurcations, Center Manifolds, and Normal Forms in Infinite Dimensional Dynamical Systems, Springer-Verlag, 2010.

[16] https://github.com/LeClaincheVega/HODMD.

[17] G.H. Golub, G.T. van Loan, Matrix Computations, John Hopkins Univ. Press, 1996.

[18] F. Takens, in: D.A. Rand, L.-S. Young (Eds.), Detecting Strange Attractors in Turbulence, in: Lecture Notes in Mathematics, Springer-Verlag, 1981, pp. 366-381.

[19] L.R. Tucker, Some mathematical notes on three-mode factor analysis, Psychometrika 3 (1966) 279-311.

[20] L. de Lathauwer, B. de Moor, J. Vandewalle, A multilinear singular value decomposition, SIAM J. Matrix Anal. Appl. 21 (2000) 1253-1278.

[21] J. Sánchez, M. Net, B. García-Archilla, C. Simó, Newton-Krylov continuation of periodic orbits for Navier-Stokes flows, J. Comput. Phys. 201 (1) (2004) 13-33.

[22] M. Dolnik, A. Zhabotinsky, I.R. Epstein, Modulated standing waves in a short reaction-diffusion system, J. Phys. Chem. 100 (1996) 6604-6607.

[23] M. Net, J. Sánchez, Symmetric periodic orbits and global dynamics of quasiperiodic flows in an $O(2)$ equivariant system: Two-dimensional thermal convection, Int. J. Bifur. Chaos Appl. Sci. Eng. 15 (12) (2005) 3953-3972.

[24] Y.A. Kuznetsov, Elements of Applied Bifurcation Theory, Springer, Berlin, 1998.

[25] I.M. Niven, H.S. Zuckerman, An Introduction To the Theory of Numbers, John Wiley and Sons, 1972.

[26] S. Le Clainche, J.M. Vega, J. Soria, Higher order dynamic mode decomposition of noisy experimental data: the flow structure of a zero-net-mass-flux jet, Exp. Thermal Fluid Sci. 88 (2017) 336-353. 